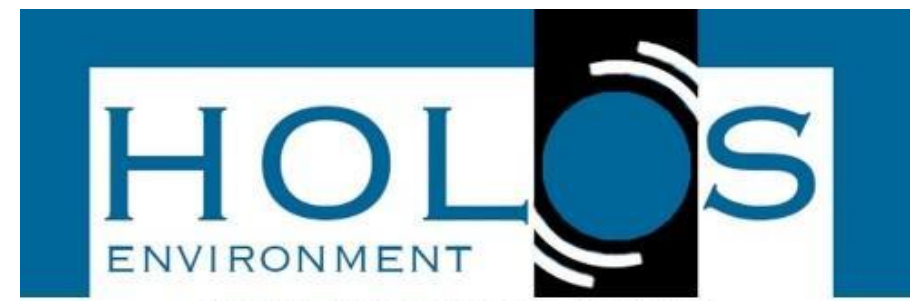

\title{
DETERMINAÇÃO DE METAIS PESADOS (FE, MN, CD, CU, CO, CR, NI, ZN E PB), MATÉRIA ORGÂNICA E CARBONO ORGÂNICO NO SEDIMENTO DE ÁREAS DE MANGUEZAL NO MUNICÍPIO DE VIGIA DE NAZARÉ, NE -PA
}

\section{DETERMINATION OF HEAVY METALS (FE, MN, CD, CU, CO, CR, NI, ZN AND PB), ORGANIC MATTER AND ORGANIC CARBON IN THE SEDIMENT OF MANGROVE AREAS IN VIGIA DE NAZARÉ CITY, NE-PA}

\author{
Anny Marysol Souza Cavalcante ${ }^{1}$; Raimundo Nonato do Espiríto Santo dos Santos ${ }^{1}$
}

Artigo recebido em: 10/08/2020 e aceito para publicação em: 19/11/2020.

DOI: http:/dx.doi.org/10.14295/holos.v21i1.12416

\begin{abstract}
Resumo: A contaminação e avaliação de metais no sedimento de ecossistemas aquáticos é um importante registrador da poluição ambiental ocorrida em locais próximos a áreas urbanas. Desse modo, o estudo investiga caracterizar e quantificar os teores de metais pesados ( $\mathrm{Fe}, \mathrm{Mn}, \mathrm{Cd}, \mathrm{Cu}, \mathrm{Co}, \mathrm{Cr}, \mathrm{Ni}, \mathrm{Zn} \mathrm{e} \mathrm{Pb}$ ), no sedimento de fundo no Furo da Laura, município de Vigia de Nazaré (PA). A área é considerada de grande importância para o complexo hidrográfico, por ser um estuário que recebe contribuição da Baía do Marajó. Foram coletadas nove amostras de sedimento que foram peneiradas na fração argila $<63 \mu \mathrm{m}$. Foi realizado a pesagem de massa de $0,5 \mathrm{~g}$ de cada ponto, para ser submetida a digestão ácida. As determinações dos metais foram realizadas por espectrometria de absorção atômica com atomização por chama, extração parcial. As determinações de matéria orgânica e carbono orgânico se utilizaram como referência o método de Walkley-Black. Apenas os resultados pra Pb (P3, P4 e P7) e Ni apresentaram valores acima do limite tolerável estipulado pelo CONAMA 454/12 Nível 1, sugerindo a contribuição antropogênica, cujas prováveis fontes de lançamentos são os efluentes domésticos e industriais do município, e também, as atividades de transporte fluvial, vazamento de combustíveis, óleos e materiais graxos. Recomenda-se que a área seja monitorada para que se possa acompanhar o comportamento desses metais, que apresentaram teores anômalos.
\end{abstract}

Palavras-chave: Amazônia. Metais. Sedimento. Absorção atômica com atomização por chama.

\begin{abstract}
Contamination and assessment of metals in the sediment of aquatic ecosystems is an important indicator of environmental contamination occurring in locations close to urban areas. The study aims to characterize and quantify the levels of heavy metals ( $\mathrm{Fe}, \mathrm{Mn}, \mathrm{Cd}, \mathrm{Cu}, \mathrm{Co}, \mathrm{Cr}, \mathrm{Ni}, \mathrm{Zn}$ and $\mathrm{Pb}$ ), at the bottom sediment at Furo da Laura, city of Vigia de Nazare (PA), the area is considered of great importance for the hydrographic complex, as it is an estuary that receives contribution from the Marajó Bay. Nine sediment samples were collected and sieved in the clay fraction $<63 \mu \mathrm{m}$. From each site, $0.5 \mathrm{~g}$ was selected to be subjected to acid digestion, and the metal determinations were performed by flame atomic absorption spectrometry with atomization, partial extraction. The determinations of organic matter and organic carbon used as a reference the Walkley-Black method. High concentrations of $\mathrm{Fe}$ and $\mathrm{Mn}$ suggest the characteristic of lateritic soils in the region, and only the concentrations for Pb (P3, P4 and P7) and Ni showed values above the tolerable limit stipulated by CONAMA Resolution No. 454/12, Level 1. However, these results are still considered concentrations below which adverse effects on the ecosystem are expected, but which suggests the anthropogenic contribution, whose probable sources of releases are domestic and industrial effluents, and also, coming from river transport activities. It is recommended that the area be monitored so that the behavior of these metals can be monitored.
\end{abstract}

Keywords: Amazon. Metals. Sediment. Atomic absorption with flame atomization.

\footnotetext{
${ }^{1}$ Universidade Federal do Pará (UFPA), Belém, PA. E-mails: anny marysol@hotmail.com, nato@ufpa.br
} 


\section{INTRODUÇÃO}

A biodiversidade do ecossistema de manguezal contempla organismos presentes nos ambientes terrestre, marinho e estuarino, devido a fauna de mangue proporcionar abrigo e criadouros naturais para diversas espécies, e por isso esse ecossistema representa ser um importante produtor primário, composto por fitomassa, biomassa e necromassa, sendo considerado a base da cadeia alimentar dos ambientes marinho e estuarino (SCHAEFFER-NOVELLI; COELHO JUNIOR, 2018).

No ecossistema de manguezal, a presença de diversos lagos de várzea é característica na região por serem formados durante as enchentes e nas vazantes dos rios. Desses lagos de várzea, são originários pequenos canais denominados "furos", definido como um canal longo e estreito (SANTOS, 1962). A movimentação de energia nos fluxos de marés nesses ambientes de várzea ocorre de forma reduzida, pelo fato da grande carga sedimentar característica do próprio ambiente, o que propicia o acúmulo natural de resíduos de origem inorgânica e orgânica (SIQUEIRA; APRILE, 2013).

A cidade de Vigia de Nazaré localiza-se em uma área de várzea, circundada pelo Furo da Laura, com duas conexões com a Baía do Marajó de aproximadamente $40 \mathrm{~km}$, recebendo influência de pequenos canais de marés ao longo de seu curso (FERNANDES, 2014). Nesse contexto, com a expansão da área urbana, ocorre a preocupação ambiental com o furo e os canais que circundam a cidade que podem sofrer com a contaminação proveniente de ação antrópica. Dentre os impactos gerados com a proximidade com a cidade, a contaminação por metais é um potencial de risco ecológico e a saúde humana, em virtude dos metais serem acumulados no meio aquático e incorporados aos sedimentos de fundo, o que torna o sedimento um componente importante do meio ambiente para o estudo da contaminação de ecossistemas aquáticos (HAN et al., 2018; RAHMAN et al., 2019).

A mobilidade dos metais no sedimento é um fator influenciado pela geologia, mineralogia, hidrologia e fatores biológicos do ambiente de estudo, além de estar relacionada com a forma física, toxidade e biodisponibilidade do metal (LEMES, 2001; ZAMBETTA 2006). Dessa forma, a análise do sedimento irá constatar todos os processos que ocorrem no ecossistema aquático por ter a capacidade de acumular compostos que o torna mais importante na avaliação do nível de contaminação do meio aquático, devido desempenhar um papel de destaque na dinâmica funcional do meio, por participar de processos internos que incluem a sedimentação, ciclagem de nutrientes e decomposição 
da matéria orgânica, podendo ainda armazenar informações sobre as formas e uso da bacia de drenagem (FAIRBRIDGE, 1989; MENDES FILHO, 2009).

Os sedimentos por serem transportados por lixiviação e erosão, são considerados como registrador da poluição ocorrida no ambiente e também por gerar uma análise de dispersão desses contaminantes, e assim ser uma fonte de observação de risco para a saúde humana e qualidade ambiental (NEVES, 1998; KABATA-PENDIAS, 2001; ALMEIDA et al., 2016). Em função deste quadro, o estudo da extração parcial de metais e da matéria orgânica do sedimento é necessário para entender a dinâmica dos metais no meio aquático. Os estudos analíticos sobre poluentes de recursos hídricos são essenciais para proporcionar informação quanto aos níveis de contaminação e ser uma ferramenta de triagem para impressão da qualidade do meio ambiente circundante.

Diante disso, o principal objetivo do presente estudo é caracterizar as concentrações dos metais: Fe, Mn, Cd, Cu, Co, Cr, Ni, Zn e Pb, no sedimento de fundo do Furo da Laura, área de mangue, na cidade de Vigia de Nazaré, com o intuito de identificar e caracterizar as fontes de metais no sedimento, bem como avaliar risco de metais pesados na área investigada e suas consequências no ambiente aquático.

\section{MATERIAL E MÉTODOS}

\section{1 Área de estudo}

O município de Vigia de Nazaré (Mapa 1) está situado a uma distância de 93 km da capital paraense. O município limita-se a oeste pela ilha de Colares e Baía do Marajó, ao sul pelo Município e Santo Antônio do Tauá, a leste pelos Municípios de São Caetano de Odivelas e Castanhal e ao norte pelo Oceano Atlântico (MOURÃO et al., 2007; FAPESPA, 2016). Localizado segundo as coordenadas geográficas: $00^{\circ} 51^{\prime} 12^{\prime \prime} \mathrm{S}$ e $48^{\circ} 08^{\prime \prime} 41^{\prime \prime} \mathrm{O}$ de Greenwich (FAPESPA, 2016).

$\mathrm{Na}$ região do município, a presença de áreas de manguezal é característica do nordeste paraense, por isso, a presença de três reservas extrativistas próximas a área de estudo, como a Reserva Extrativista Marinha Mocapajuba, a Reserva Extrativista Marinha de São João da Ponta e a Reserva Extrativista Marinha Mãe Grande de Curuçá, demonstram a preocupação com a biodiversidade de áreas de mangue.

No perfil geomorfológico da região, a presença de áreas de mangue é quantificada por Souza Filho (2005) com uma área de $842,81 \mathrm{~km}^{2}$, estendendo-se da Baía do Marajó até Pirabas, com uma planície costeira e restrita com menos de $2 \mathrm{~km}$ de largura, além de 
canais estuarinos com aproximadamente $60 \mathrm{~km}$. O sedimento terciário presente é característico da Formação Barreiras, com a presença de baixadas semi litorâneas e terraços de várzea, sujeito a alterações de ondas e correntes de marés (SOUZA FILHO, 2005; PACHECO, 2011). A constituição do solo de Vigia é composta por Latossolo Amarelo distrófico textura média, Areias Quartzonas distróficas, Glei Pouco Úmido distrófico com textura e argilosa, e PodzolHidromófico (SANTOS, 2012; PACHECO, 2011; FERREIRA, 2015). O relevo é constituído de baixos platôs pediplanados e rebaixados, em que estão presentes os níveis mais baixos e inseridos na unidade morfoestrutural que constitui 0 Planalto Rebaixado da Amazônia (PACHECO, 2011).

Na região amazônica, a presença de "furos", um termo popularmente amazônico, é uma das características da região e são considerados de grande importância para o complexo hidrográfico, por ser um estuário que recebe contribuição de rios grandes e pequenos (SANTOS, 1962; ALVES, 2015). Segundo Alves (2015), os furos são um canal de drenagem que liga um rio a outro, um rio a um lago ou um rio a ele mesmo, sendo dependente da dinâmica fluvial dessa conexão, por isso, os furos são fundamentais por servirem de aporte fluvial nas época de cheia para outros lagos da região, e durante a vazante, inverte-se a direção da correnteza e corre a liberação das águas para o canal ou rio principal através destes furos.

Nesse contexto, Furo da Laura recebe influência de pequenos canais e das correntes de mares provenientes da Baía do Marajó que servem como mecanismo de mistura entre as águas doces dos rios e as águas salgadas do ambiente marinho. Além disso, fatores naturais como chuvas, correntes, o transporte e deposição de sedimentos, influenciam nas interações entre os contaminantes, a água e sedimento, assim como aumentam a distribuição de contaminantes existentes na área (CORRÊA, 2005; FERNANDES, 2014).

\subsection{Procedimento de campo}

A coleta foi realizada em setembro de 2018, no período de seca. Foram escolhidos nove pontos para coleta de sedimento de fundo para se verificar a influência da descarga de resíduos de esgotos urbanos e industriais provenientes da cidade de Vigia de Nazaré, no Furo da Laura. Em cada ponto, nove amostras compostas de sedimentos foram coletadas com o auxílio de uma draga de Eckman. O método de coleta das amostras de sedimento seguiu a metodologia efetuada por Berrêdo (2006), sendo adaptada para o sedimento do local de estudo. Os pontos de amostragem foram devidamente fechados, identificados, acondicionados em caixa térmica para proteção e conservação da matéria 
orgânica e para se evitar a contaminação e atividade biológica. A localização geográfica de cada local de amostra foi obtida utilizando um GPS. Posteriormente foram feitas as análises em laboratório (Mapa 2).

Mapa 1 - Localização do Município de Vigia

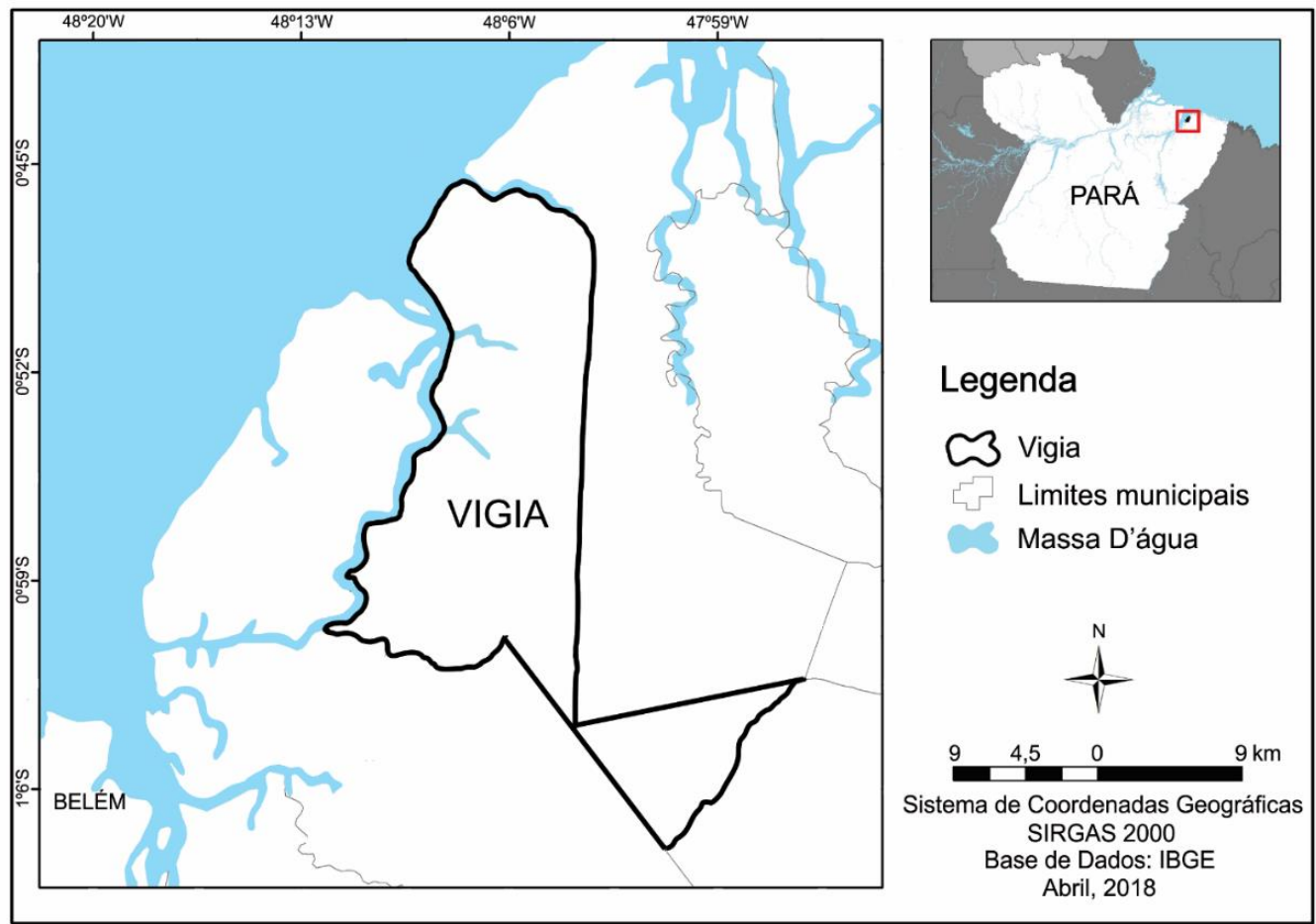

Mapa 2 - Localização dos pontos de coleta de sedimento

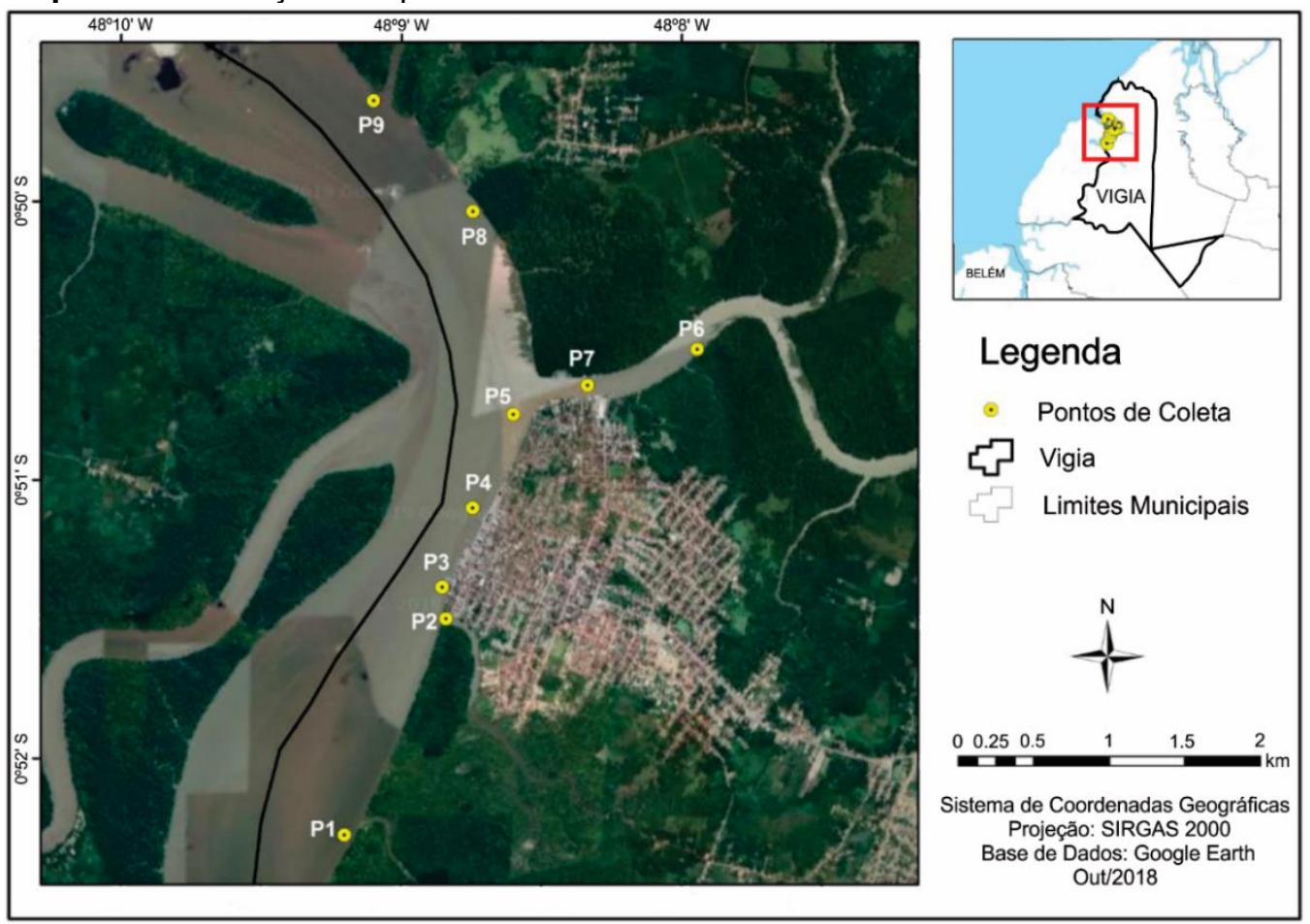

\subsection{Procedimentos em laboratório}

Para cada amostra dos nove pontos de coleta de sedimento de fundo, foram retira- 
dos $1.000 \mathrm{~mL}$ de cada amostra, e acrescentado mais $1.000 \mathrm{~mL}$ de água destilada em um Becker de $2.000 \mathrm{~mL}$. As amostras foram submetidas a um processo de amostragem na técnica de separação da fração argila. Um banho de ultra-som no modelo Embrasol TEC, LimpSonic, LS-55DA-2/X AQUEC - $33 \mathrm{kHz}$, por 12 minutos, foi utilizado para homogeneização das suspensões do sedimento. Após, o sobrenadante foi retirado e realizado o peneiramento de $63 \mathrm{~mm} / \mu \mathrm{m}(<0,063 \mathrm{~mm})$ de malha. Após 30 minutos de repouso, as subamostras de cada ponto da coleta, foram separadas em 20 tubos de $50 \mathrm{~mL}$, com $45 \mathrm{~mL}$ de amostra. Cada tubo foi pesado, para estar na medição de $66 \mathrm{~g}$ cada, e então ir à centrifugação por 5 minutos. Em seguida, as amostras foram separadas em cápsulas de porcelana, para identificação e descrição dos materiais da fração argila. Trata-se de uma adaptação do método utilizado por Berrêdo (2006).

\subsection{Determinação da análise de metais no sedimento}

Para determinar a concentração de cada elemento foi utilizada a técnica de análise química para a extração parcial de teores de metais em sedimento de fundo, a espectometria de absorção atômica por chama segundo Evans et al. (2014). Foi utilizado para essa técnica o modelo ICE 3500, marca Thermo, para ser possível determinar a concentração de metais na amostra.

Os metais pesados analisados: Ferro ( $\mathrm{Fe})$, Manganês $(\mathrm{Mn})$, Cádmio $(\mathrm{Cd})$, Cobre (Cu), Níquel ( $\mathrm{Ni}$ ), Zinco ( $\mathrm{Zn}$ ) e Chumbo (Pb) foram determinados por digestão ácida em que consiste em utilizar $0,5 \mathrm{~g}$ da fração fina $(<0,063 \mathrm{~mm})$ por fusão e combustão, composta pelos reagentes $\left(\mathrm{HNO}_{3}\right.$ e $\left.\mathrm{HCl}\right)$. A fusão se baseia na decomposição de materiais inorgânicos que ocorre em elevada temperatura, enquanto a combustão é a decomposição de materiais orgânicos em que a queima da amostra é realizada pelo oxigênio do ar que atua como oxidante.

As amostras foram colocadas em cadinho de teflon para o processo. Sendo então, adicionado $5 \mathrm{~mL}$ de $\mathrm{HNO}_{3}$ concentrado e $5 \mathrm{~mL}$ de $\mathrm{HCl}$ concentrado nas amostras. Após, foi realizado a evaporação dos reagentes na temperatura de $90^{\circ} \mathrm{C}$ até a secura total da amostra. Em seguida, as amostras recebem $1 \mathrm{~mL}$ de $\mathrm{HNO}_{3}$ a $2 \%$. Ocorre novamente $\mathrm{O}$ aquecimento para evaporação do reagente, para finalizar adicionando $5 \mathrm{~mL}$ de $\mathrm{HNO}_{3}$ a $2 \%$. O processo se conclui com as amostras sendo filtradas para obtenção de uma solução de $25 \mathrm{~mL}$ sem resíduo sólido. 


\subsection{Determinação do teor de carbono e matéria orgânica e carbono orgânico}

A análise química para determinação de teor de matéria orgânica e carbono de solo foi utilizada como referência o método de Walkley-Black. Para determinação de carbono orgânico (C.O\%) foram pesadas amostras secas de sedimento com $0,1 \mathrm{~g}$ de cada amostra da fração argila dos pontos de coleta para ser oxidada pela ação de Dicromato de Potássio e Ácido Sulfúrico, $10 \mathrm{ml}$ cada. Após a reação, é adicionado $3 \mathrm{ml}$ de Ácido Fosfórico concentrado, e posteriormente é acrescentado $50 \mathrm{ml}$ de água deionizada. Usa-se a difenilamina como indicador. Para a determinação de matéria orgânica (M. O. \%), é multiplicado a porcentagem de carbono orgânico pelo fator de 1,725.

\subsection{Valores orientadores de qualidade de sedimento utilizados no estudo}

Devido à falta de estudos atuais relacionados a contaminação de sedimento de fundo desse recurso hídrico, os resultados obtidos no trabalho foram comparados com os índices de referência da resolução CONAMA 454/12 para análise dos metais $\mathrm{Pb}, \mathrm{Cu}, \mathrm{Cr}$, Ni e Zn, que define identificação de concentrações de substâncias químicas e gestão ambiental das fontes, pontuais e difusas de poluição hídrica, para a redução e a prevenção da poluição dos sedimentos. A resolução estabelece a caracterização química do sedimento para determinar as concentrações das substâncias poluentes contidas na fração residual das amostras. Após essa caracterização, são definidos níveis de classificação para definir o critério de qualidade, a partir de dois níveis, nível 1: limiar abaixo do qual prevê-se baixa probabilidade de efeitos adversos à biota; nível 2: limiar acima do qual prevê-se um provável efeito adverso à biota (CONAMA, 2012).

A resolução CONAMA 420/09 foi utilizada para análise dos metais $\mathrm{Cd}$ e Co, e foi estabelece como critério de valores orientadores de qualidade (VQR) do solo para gerenciamento ambiental de áreas contaminadas por essas substâncias em decorrência de atividades antrópicas.

Além das legislações, também foi comparado com valores normalizados da crosta terrestre e áreas de mangue com característica sedimentológica da Formação Barreiras, presente na região de estudo.

\section{RESULTADOS E DISCUSSÃO}

O objetivo geral do estudo foi caracterizar as concentrações dos metais no sedimento 
de fundo do Furo da Laura, área de mangue no município de Vigia. Os resultados da análise de extração parcial de metais pesados no sedimento são caracterizados por alguns pontos se encontrarem com a concentração de metais acima do permitido da legislação CONAMA 454/12 e CONAMA 420/09. Esses resultados indicam uma contaminação nos pontos em frente ao município de Vigia (P3, P4 e P7) para $\mathrm{Pb}$ e Ni, e em todos os pontos de amostragem de sedimento foi obtido os maiores resultados para Fe e Mn (Tabela 1).

A partir do método de espectrometria de absorção atômica, foi possível observar que os valores descritivos e o padrão de distribuição dos metais apresentaram uma concentração decrescente de ordem $\mathrm{Fe}>\mathrm{Mn}>\mathrm{Zn}>\mathrm{Cr}>\mathrm{Pb}>\mathrm{Co}>\mathrm{Ni}>\mathrm{Cu}$. Além de ser possível comparar com outros valores de referência de áreas de manguezal e com valores da Crosta Terrestre (Tabela 2).

Tabela 1 - Concentração de metais pesados das amostras de sedimentos em $\mathrm{mg} \mathrm{kg}^{-1}$, determinada por espectrometria de absorção atômica, e concentração de matéria orgânica (M. O.) e carbono orgânico (C. O.) e diretrizes da legislação brasileira

CONAMA no
454/2012.

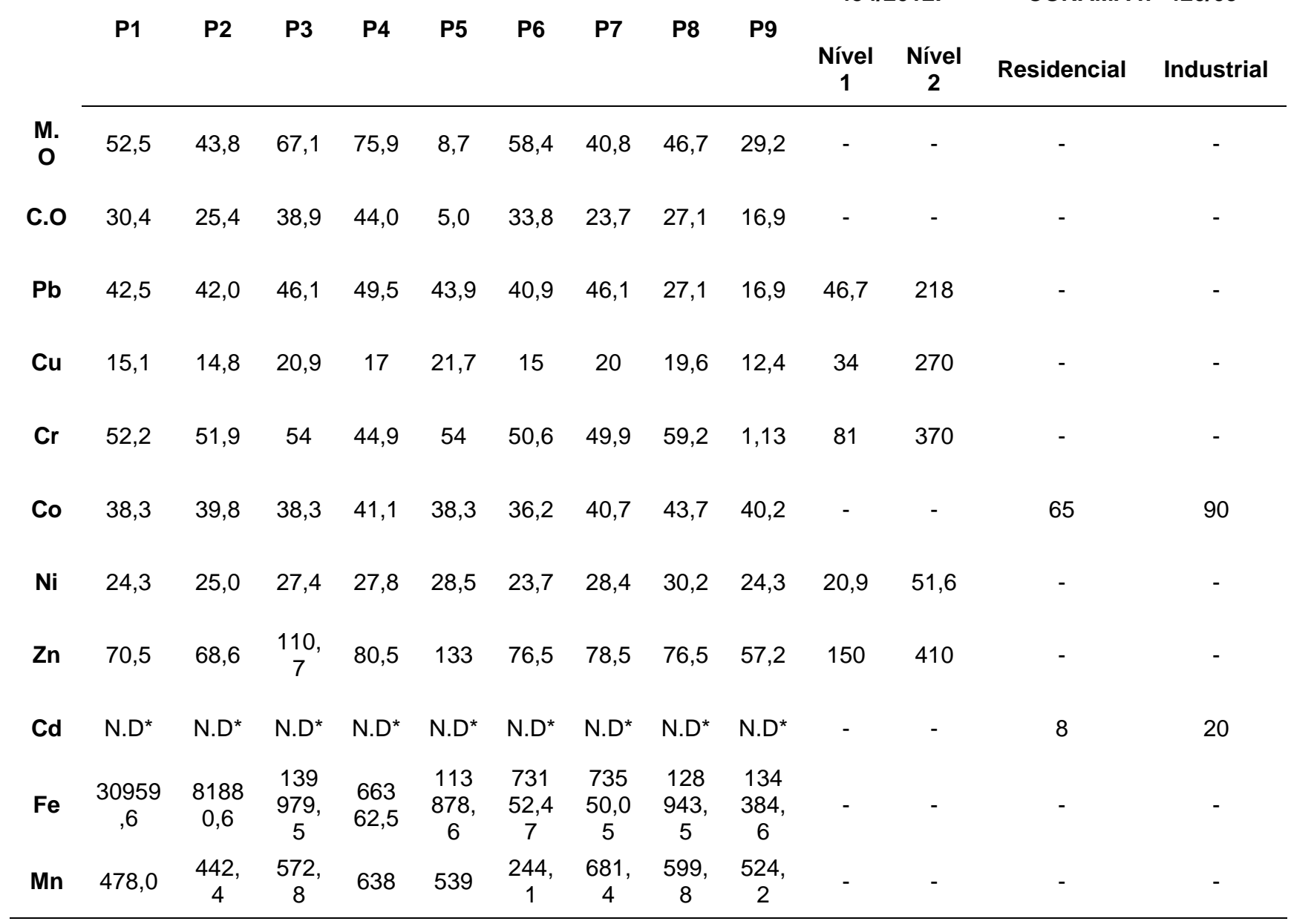

Não determinado* 
Tabela 2- Concentração dos metais pesados nos sedimentos do Furo da Laura e sedimentos de áreas de manguezal e com a Crosta Terrestre, expressos em mg kg-1

\begin{tabular}{ccccc}
\hline Metais & Furo da Laura $^{1}$ & Marapanim $^{2}$ & Bragança $^{2}$ & Crosta terrestre $^{3}$ \\
\hline $\mathrm{Fe}$ & 93676,8 & 6,51 & 5,20 & 56000 \\
$\mathrm{Mn}$ & 524,4 & 0,03 & - & 950 \\
$\mathrm{Zn}$ & 83,6 & 66 & 52 & 70 \\
$\mathrm{Ni}$ & 26,6 & 22 & 14 & 75 \\
$\mathrm{Co}$ & 39,6 & 8 & 12,3 & 25 \\
$\mathrm{Cr}$ & 46,4 & 10,7 & 78 & 100 \\
$\mathrm{Cu}$ & 17,4 & 14,3 & 30 & 55 \\
$\mathrm{~Pb}$ & 44,0 & 60,6 & 12,5 \\
\hline
\end{tabular}

${ }^{1}$ Média dos valores obtidos no presente estudo;

2 Manguezais do nordeste do estado do Pará - Berrêdo (2006);

${ }^{3}$ Crosta terrestre - Krauskopf (1972b).

A concentração de Cd obteve-se abaixo do limite de detecção de $1 \mu \mathrm{g} \mathrm{L}^{-1}$, por isso os resultados sugerem que não existem teores significantes de $\mathrm{Cd}$ no sedimento de fundo do Furo da Laura. Os resultados obtidos são semelhantes do estudo de Tomazelli (2003) nas bacias hidrográficas de São Paulo, que constatou concentrações de Cd no sedimento inferiores ao limite de detecção. A disponibilidade do metal depende da vazão dos rios e dos parâmetros físicos químico da água, além da quantidade de material em suspensão e outros parâmetros do ambiente (TOMAZELLI, 2003).

As concentrações de Cu determinadas no sedimento do Furo da Laura são similares aos valores encontrados por Oliveira et al. (2015), no sedimento de fundo da Baía do Guajará (PA), e por Siqueira e Aprile (2013) na Baía do rio Aurá (PA), os estudos concluem que as concentrações retratam uma condição natural do elemento. Além disso, a baixa concentração de $\mathrm{Cu}$ associada a alta concentração de matéria orgânica no sedimento do Furo da Laura, vão em contrapartida ao estudo de Zhang et al. (2018), em que sugerem que altas concentrações de matéria orgânica aumentam a mobilidade de Cu no sedimento. Ainda assim, a maior concentração de matéria orgânica no P3 $\left(67,1 \mathrm{mg} \mathrm{kg}^{-1}\right)$ mostrou a maior concentração de $\mathrm{Cu}\left(20,9 \mathrm{mg} \mathrm{kg}^{-1}\right)$, o que pode indicar uma liberação de Cu através do processo de sedimentação e sua adsorção na superfície do sedimento (KRAUSKOPF,1972b).

Nas concentrações de Ni no sedimento, valores acima da legislação CONAMA no 454/2012 (51,6 mg kg-1) Nível 2, são considerados de grande toxidade e que causam impactos adversos nas funções do sedimento, além de problemas ambientais e a bioacumulação para as cadeias alimentares (RINKLEBE; SHAHEEN, 2017). O valor mais alto de Ni encontrado no sedimento foi observado no P8 (30,2 $\left.\mathrm{mg} \mathrm{kg}^{-1}\right)$. Em todos os pontos os valores registrados se encontram levemente acima do estabelecido na legislação CONAMA no 454/2012, nível 1 (20,9 mg kg-1). Contudo, a concentração de Ni encontrada 
ainda não sugere que o elemento cause impactos adversos no ecossistema da região. Nesse contexto, valores similares $\left(24 \mathrm{mg} \mathrm{kg}^{-1}\right)$ foi encontrado por Carvalho (2002) no trabalho sobre a caracterização geoquímica de sedimento de fundo sob influência do lançamento de esgoto na Orla de Icoaraci (PA). Assim como, valores similares observados no sedimento de fundo da Baía do Guajará (PA) por Oliveira et al. (2015) e sugerem que os valores obtidos indicam contribuição naturais dos elementos. Desse modo, é possível verificar que o teor de $\mathrm{Ni}$, ainda não sugere que a região está impactada pelo elemento, sendo que é possível confirmar que existe uma forte influência da Formação Barreiras devido à própria composição da crosta continental e das rochas sedimentares serem ligeiramente enriquecidas com Ni (KRAUSKOPF, 1972b; BERRÊDO, 2006).

Os valores determinados para Co no sedimento do Furo da Laura são maiores que os valores encontrados por Berrêdo (2006) em áreas de manguezal e maiores que os valores definidos por Krauskopf (1972b) na crosta terrestre. No estudo de Siqueira et al. (2006), para análise de elementos traços no sedimento da Plataforma Continental Amazônica sob influência da descarga do rio Amazonas, encontraram teores de Co mínimos de $14,2 \mathrm{mg} \mathrm{kg}^{-1}$, e teores máximos de $48,7 \mathrm{mg} \mathrm{kg}^{-1}$, maiores que o resultados obtidos no presente estudo. Para Siqueira et al. (2006), a presença de Co no ambiente é considerada como de ocorrência natural, não sendo influência de origem poluidora. A presença de Co também é justificada por Alves e Della Rosa (2003), em que o metal ocorre devido a queima de combustíveis fosseis, o uso de biossílidos e fertilizantes fosfatados, mineração e fundição de minérios e de processos industriais que contenham o metal.

As concentrações e Pb no ambiente se mostram diferentes nas unidades litológicas, pois dependendo da composição e da abundância dos minerais presentes no sedimento, 0 elemento possui maior ou menor prevalência (CHAKRABORTY; CHAKRABORTY; NATH, 2015). Além da origem natural, a liberação de $\mathrm{Pb}$ ocorre principalmente pelas atividades de mineração, através da combustão de carvão e gasolina, produção de ferro, aço e cimento, fundição de metais não ferrosos, poeiras, minério de chumbo doméstico, exaustão de veículos e pó metalúrgico, e junto com o intemperismo, contribui com a contaminação para o meio ambiente (WANG et al., 2017).

As maiores concentrações de $\mathrm{Pb}$ nos resultados obtidos ocorreram nos pontos localizados em frente ao município de Vigia, P3 (46,1 mg kg-1), P4 (49,5 mg kg-1) e P7 (46,1 $\mathrm{mg} \mathrm{kg}^{-1}$ ). O P4 é o único ponto que se encontra acima do valor limiar de probabilidade de efeitos adversos à biota pela legislação CONAMA no 454/2012, Nível 1 (46,7 mg kg-1). Essa prevalência pode ser induzida pela localização do ponto estar em frente de uma indústria 
pesqueira. Contudo, todos os pontos se encontram com valores próximos ao estabelecido no Nível 1 do CONAMA no 454/2012, o que indica o princípio de contaminação pelo metal.

Devido a área de estudo ser de grande movimentação de embarcações pesqueiras, Silva (2016), relata que a presença de Pb no sedimento é originário dos combustíveis que contém tetraetilo e tetrametilo de chumbo que funcionam como anti-deteriorantes. $E$ justamente, por causa da cidade ser considerada um dos maiores polos pesqueiros do Estado do Pará, a presença da pesca artesanal e industrial é de grande importância na região, o despejo de combustível na área de estudo provavelmente representa uma grande contribuição para a contaminação de $\mathrm{Pb}$ no sedimento. Além disso, próprio ambiente favorece a contribuição do metal no sedimento, sendo o Pb também proveniente da crosta terrestre e de áreas de mangue (KRAUSKOPF, 1972b; BERRÊDO, 2006).

As concentrações de Cr se apresentaram abaixo do limite determinado na legislação $\left(81 \mathrm{mg} \mathrm{kg}^{-1}\right.$ ) CONAMA nำ454/2012. Ainda assim, a presença desse metal nas análises indica que o ambiente recebe a descarga de esgoto doméstico e industrial (LEMES, 2001; OLIVEIRA et al., 2014). Contudo, existe a contribuição do próprio ambiente enriquecido com o elemento, devido aos processos sedimentares existentes, sendo o ambiente enriquecido com a contribuição da crosta terrestre e outras áreas de mangue (KRAUSKOPF, 1972b; BERRÊDO, 2006). Berrêdo (2006), em estudo no estuário do rio Marapanim no sedimento terciário da Formação Barreiras, verifica a presença de Cr como um metal característico do processo de intemperismo da Formação Barreiras. Desse modo, é justificável o teor de Cr encontrado no sedimento de fundo do Furo da Laura como parte da geologia da Formação Barreiras.

De acordo com Holanda (2010), no seu estudo sobre o comportamento de metais no sedimento de fundo da Baía do Guajará e na Baía do Marajó, observa que a geologia da Formação Barreiras faz com que se encontre teores de Cr com uma média de até $102 \mathrm{mg}$ $\mathrm{kg}^{-1}$ nessa região, sugerindo que o metal possui uma leve tendência de se concentrar onde existe maior teor de matéria orgânica. Logo, apesar do baixo teor de $\mathrm{Cr}$ encontrado no sedimento de fundo do Furo da Laura, embora não ultrapassando o limite permitido de análise da qualidade dos sedimentos, é justificável pelo alto teor de matéria orgânica.

O Zn é um metal necessário para atividade do ecossistema e para o seu funcionamento dos processos fisiológicos e metabólicos, porém, como todo metal em grandes concentrações, podem tornar-se tóxicos (BORAH et al., 2018). As concentrações de $\mathrm{Zn}$ encontradas no sedimento do Furo da Laura não se encontram acima da legislação CONAMA nำ 454/2012 (150 mg kg-1). Ainda assim, as altas concentrações determinadas 
no P3 $\left(110,78 \mathrm{mg} \mathrm{kg}^{-1}\right)$ e no P5 $\left(133,03 \mathrm{mg} \mathrm{kg}^{-1}\right)$, podem indicar a forte influência das atividades antrópicas. Esse fato é ratificado por Milesi et al. (2008) por verificar que as atividades industriais e a proximidade com os perímetros urbanos são os principais efluentes para a contaminação de água e solo por Zn, pelo fato de o metal possuir afinidade de acumulação com o sedimento. Além disso, existe a contribuição do metal por processos sedimentares provenientes da Formação Barreiras e da Crosta terrestre, nas próprias rochas sedimentares (KRAUSKOPF, 1972b; BERRÊDO, 2006)

O Fe é um metal essencial para o ecossistema por possuir micronutrientes reguladores para ocorrência de vários processos biológicos e biogeoquímicos, por isso, seus teores são maiores comparados aos outros metais (KAUSHIK et al.,2009; ARAÚJO et al., 2017). No sedimento do Furo da Laura, as maiores concentrações médias determinadas foram do Fe (93676,8 $\left.\mathrm{mg} \mathrm{kg}^{-1}\right)$, seguido pelos valores de $\mathrm{Mn}\left(524,4 \mathrm{mg} \mathrm{kg}^{-1}\right)$.

Siqueira e Aprile (2013), no estudo sobre o comportamento geoquímico de Fe e Mn no sedimento da Bacia do rio Aurá (PA), encontrou resultados similares ao do presente estudo para os dois elementos. A facilidade de associação de Fe com os argilo minerais propicia a precipitação do metal. Assim como o Mn é incorporado a determinados minerais como a biotita (SIQUEIRA; APRILE, 2013).

A alta concentração de Fe e Mn encontrada no sedimento de fundo do Furo da Laura é associada com as características geológicas dos sedimentos da Formação Barreiras segundo Sartori et al. (2005) e Berrêdo et al. (2008), que consideraram a influência da ação de correntes marítimas provenientes da Baía do Marajó como contribuição da precipitação e deposição dos dois metais no sedimento por conter óxidos de ferro (hematita, goetita), óxidos de alumínio (gbsita) e argila de baixa atividade (argilo minerais).

Além da contribuição geológica, Kanbar et al. (2017) associam a presença de $\mathrm{Fe}$ devido os resíduos industriais, em que é possível encontrar fontes de ferro, tais como minério de ferro, ferro fundido, e as fases do elemento, como de óxido de ferro (II e III), sulfeto de ferro. O elevado teor de Fe no sedimento do Furo da Laura pode ser justificado devido a localização dos pontos de amostragem estarem em área portuária e aréa de despejo de esgoto proveniente do município de Vigia, sendo necessários estudos mais profundos para determinar as fontes de Fe pressente na área.

A distribuição da concentração de matéria orgânica nos pontos de coleta do Furo da Laura apresentou uma alta concentração nos pontos P1, P3, P4 e P6 que estão próximos do porto do município de Vigia de Nazaré. O inverso aconteceu, nos pontos P2, P5, P7, P8 e P9, apresentaram uma diminuição de valores, que apesar de estarem próximos ao porto, 
estão localizados em um dos canais do Furo da Laura, logo, de grande movimentação de águas e dinâmica do solo.

A posição dos pontos do estudo relacionado com a baixa concentração de matéria orgânica, confirma os estudos de Lima et al. (2004) e Siqueira et al. (2003), em que relatam a baixa concentração como resultado da deposição e ressuspensão do material particulado, e também pelo fato de ser uma área mais oxigenada e de maior movimentação do sedimento.

Berrêdo (2006), justificam a alta concentração de matéria orgânica em áreas de manguezal devido a presença de síltico-argilosos que são ricos em matéria orgânica, como também pela grande ocorrência de atividade biológica e dos processos geoquímicos. Para Casado-Martínez et al. (2006), em lugares de baixa energia, como áreas portuárias, o acumulo de contaminantes e matéria orgânica tendem a se a depositar no sedimento

Na matriz de correlação (Tabela 3), foi constatado seis correlações positivas entre os seguintes metais: $\mathrm{Pb}$ x Mn, $\mathrm{Pb}$ x Ni, Cu x Ni, Cu x Zn, Mn x Ni e Mn x Co. No Dendograma de Pearson, a melhor afinidade de agrupamento e ligação de proximidade dos metais $\mathrm{Pb}$, $\mathrm{Cu}, \mathrm{Ni}, \mathrm{Zn}, \mathrm{Mn}$ e Co (Figura 1).

Tabela 3 - Valores de correlação dos metais pesados dos sedimentos de fundo do Furo da Laura, matéria orgânica (M.O.) e carbono orgânico (C.O). Os valores em negrito são os que possuem uma maior correlação entre os metais

\begin{tabular}{lrrrrrrrrrr}
\hline & \multicolumn{1}{c}{$\mathrm{Pb}$} & \multicolumn{1}{c}{$\mathrm{Cr}$} & $\mathrm{Mn}$ & $\mathrm{Fe}$ & $\mathrm{Co}$ & $\mathrm{Cu}$ & $\mathrm{Ni}$ & $\mathrm{Zn}$ & M. O. & C. O. \\
\hline $\mathrm{Pb}$ & 1 & & & & & & & & & \\
$\mathrm{Cr}$ & 0,47 & 1 & & & & & & & & \\
$\mathrm{Mn}$ & $\mathbf{0 , 7 1}$ & 0,00 & 1 & & & & & & \\
$\mathrm{Fe}$ & $-0,04$ & $-0,28$ & 0,22 & 1 & & & & & \\
$\mathrm{Co}$ & 0,45 & $-0,05$ & $\mathbf{0 , 7 1}$ & 0,27 & 1 & & & & & \\
$\mathrm{Cu}$ & 0,64 & 0,63 & 0,52 & 0,31 & 0,15 & 1 & & & & \\
$\mathrm{Ni}$ & $\mathbf{0 , 7 5}$ & 0,44 & $\mathbf{0 , 7 5}$ & 0,38 & 0,65 & $\mathbf{0 , 8 4}$ & 1 & & & \\
$\mathrm{Zn}$ & 0,35 & 0,45 & 0,17 & 0,32 & $-0,29$ & $\mathbf{0 , 8 1}$ & 0,49 & 1 & & \\
$\mathrm{M}$. O. & 0,46 & 0,24 & $-0,02$ & $-0,30$ & 0,00 & $-0,12$ & $-0,09$ & $-0,27$ & 1 & \\
$\mathrm{C}$. O. & 0,46 & 0,24 & $-0,02$ & $-0,30$ & 0,00 & $-0,12$ & $-0,09$ & $-0,27$ & 1 & 1 \\
\hline
\end{tabular}

${ }^{*}$ Nível de significância a 5\%.

Harbison (1986) sugere que a correlação positiva de Mn com Pb, Ni e Co, ocorre devido a oxidação de Fe e hidróxidos de manganês através da adsorção e co-preciptação com outros metais, e assim podem resultar na remobilização dos metais na interação sedimento/água.

Todos os metais não apresentaram correção positiva com a matéria orgânica. Esse fato é relatado por Oliveira et al. (2015) que observam que possivelmente a matéria orgânica existente não possui propriedades complexantes satisfatórias de modo a fixar os 
metais. Além disso, Oliveira et al. (2015) e Tomazelli (2003), constatam que a deficiência desses grupos complexantes pode ser por causa de restos de vegetação, detritos de animais e da carga orgânica lançada no rio através de esgotos urbanos, o que causa a baixa capacidade de absorção e biodisponibilidade dos metais.

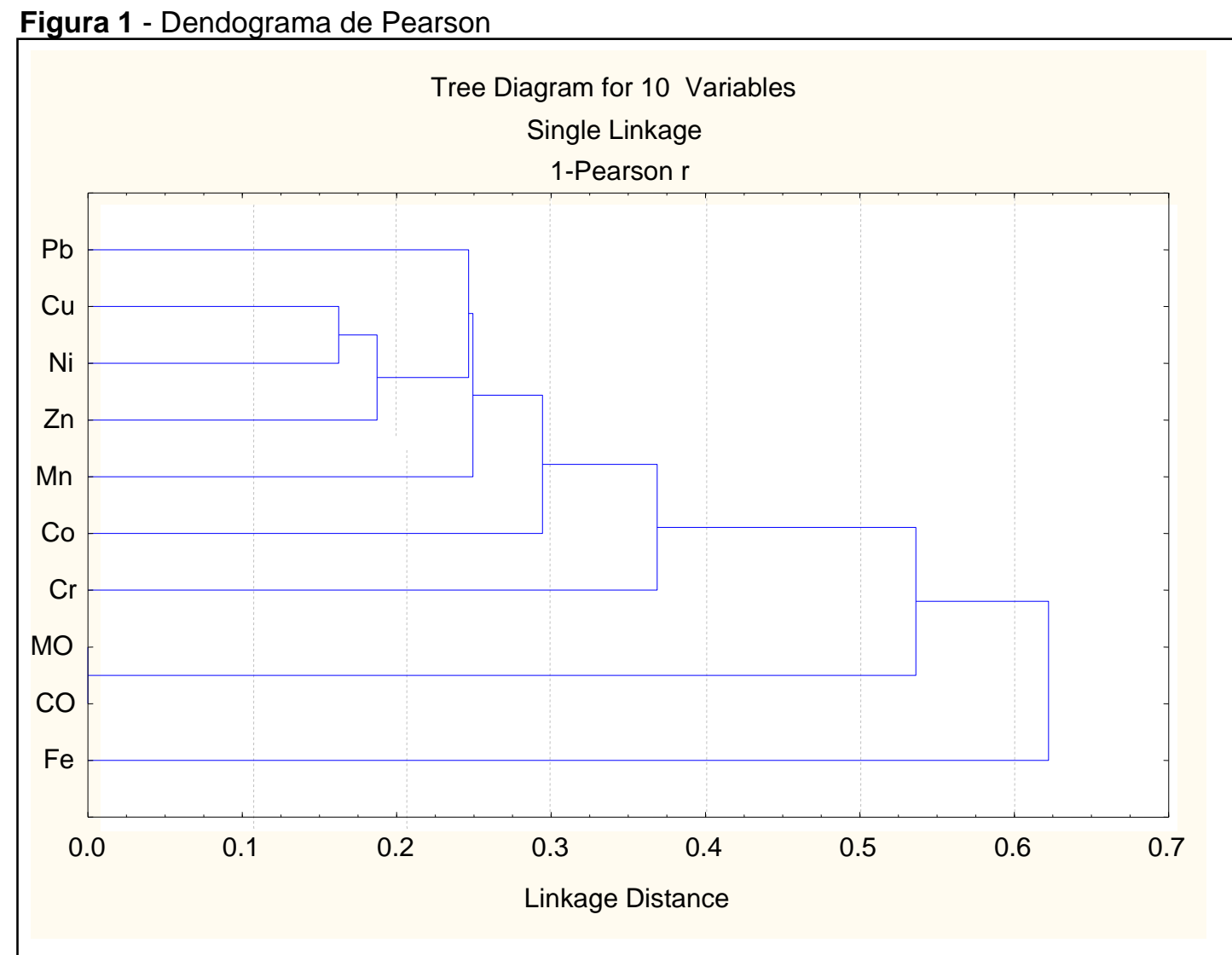

Conforme Zambetta (2006), o tamanho do grão do sedimento é um dos fatores que interferem na deposição dos metais, e consequentemente, na correlação positiva. Rubio et al. (2000) verificaram que partículas inferiores a $63 \mu \mathrm{m}$, o metal tende a estar em maior concentração. Ainda assim, a concentração de metais no sedimento não poder ser considerada apenas pela granulometria, sendo necessário observar fatores como a composição química, a mineralogia do substrato e processos físico-químicos, que também interferem na distribuição dos metais e na diferença de concentrações (MARCHAND et al., 2006; APRILE; BOUVY, 2008).

A avaliação da contaminação de metais em sedimentos de estuários é de difícil realização (RUBIO et al., 2000), assim não é possível afirmar com precisão que as correlações positivas de Pearson podem indicar ligação dos metais para uma contaminação natural ou antropogênica, mas é sugerido que existe a contribuição da 
geologia da área de estudo e a contribuição do município de Vigia dos despejos urbanos e industriais, para as altas concentrações de metais determinados.

\section{CONCLUSÃO}

O presente estudo quantificou e avaliou o enriquecimento natural, de fontes antropogênicas, os níveis de contaminação e toxidades dos metais (Fe, Mn, Zn, Cr, Pb, Co, $\mathrm{Ni}, \mathrm{Cu}$ ) em amostras de sedimento fundo no Furo da Laura. Os valores revelaram que na área de estudo as concentrações de $\mathrm{Pb}$ (P3, P4 e P7) e Ni, estão acima dos valores da legislação CONAMA nº 454/2012, nível 1, ou seja, ainda são concentrações consideradas abaixo do qual se prevê efeitos adversos no ecossistema.

As altas concentrações de Fe e Mn encontradas no sedimento é motivado pelas características dos solos lateríticos da região, que são submetidos a processos intempéricos e são lixiviados para dentro do corpo hídrico. Devido à proximidade com a cidade, é considerado a contribuição antropogênica, cujas fontes de lançamentos são os efluentes domésticos e industriais da cidade de Vigia de Nazaré e também, as atividades de transporte fluvial, vazamento de combustíveis, óleos e materiais graxos.

Os teores de matéria orgânica em todos os pontos mostraram elevadas concentrações. Os maiores teores de matéria orgânica (P3, P4) sugerem que que são locais de baixa energia, por isso ocorreu o maior acumulo nesses locais. O menor teor de matéria orgânica (P5), sugere que a localização do ponto de coleta na entrada de um dos canais do Furo da Laura, onde tem-se um fluxo energético grande, que evita o acumulo de matéria orgânica nesses locais.

Recomenda-se que a área seja monitorada para que se possa acompanhar o comportamento desses metais. Os resultados expandem o conhecimento sobre a interação entre metais e sedimento, e permite que se possa acompanhar no futuro o comportamento desses metais no Furo da Laura.

\section{AGRADECIMENTOS}

Ao Laboratório de Sedimentologia e Minerais Pesados do Instituto de Geociências da Universidade Federal do Pará (UFPA), e ao Museu Paraense Emilio Goeldi. 


\section{REFERÊNCIAS}

ALMEIDA, I. L.S. et al. Suitable extraction of soils and sediments for mercury species and determination combined with the cold vapor generation atomic absorption spectrometry technique. Microchemical Journal, Brasil, v. 124, n. 09, p.320-330, jan. 2016. https://doi.org/10.1016/j.microc.2015.09.007

ALVES, A. N. L.; DELLA ROSA, Henrique Vicente. Exposição ocupacional ao cobalto: aspectos toxicológicos. Revista Brasileira de Ciências Farmacêuticas, São Paulo, v. 39, n. 2, p.129-139, 2003. https://doi.org/10.1590/S1516-93322003000200003

ALVES, N. de S. Mapeamento hidromorfodinâmico do complexo fluvial de anavilhanas: contribuição aos estudos de geomorfologia fluvial de rios amazônicos. Produção acadêmica. São Paulo, 1741,45, 2015.

APRILE, F.M.; BOUVY, M. Distribution and Enrichment of Heavy Metals in Sediments at the Tapacurá River Basin, North Eastern Brazil, Braz. J. Aquat. Sci. Technol., v. 12, n. 1, p. 1-8, 2008. https://doi.org/10.14210/bjast.v12n1.p1-8

ARAÚJO, Amanda Dias et al. Avaliação geoquímica ambiental do garimpo areinha: estudo da concentração e distribuição de metais pesados nos sedimentos e os danos à saúde

humana. Revista Brasileira de Geografia Médica e da Saúde, Uberlândia, v. 26, n. 13, p. 98113, dez. 2017. https://doi.org/10.14393/132608

BERRÊDO, José Francisco. Geoquímica dos sedimentos de manguezais no nordeste do estado do Pará: um exemplo do estuário do rio Marapanim. 2006. 198 f. Tese (Doutorado) Curso de Geoquímica e Petrologia, Centro de Geociências, Universidade Federal do Pará, Belém, 2006.

BRASIL. Resolução no 420, de 28 de dezembro de 2009. Brasília, DF: Conselho Nacional do Meio Ambiente - CONAMA., 30 dez. 2009. p. 81-84. Disponível em: http://www.mma.gov.br/port/conama/legiabre.cfm?codlegi=620. Acesso em: 12 jan. 2018.

BRASIL. Resolução no 454, de 01 de novembro de 2012 - Estabelece as diretrizes gerais e os procedimentos referenciais para o gerenciamento do material a ser dragado em águas sob jurisdição nacional. Brasília, DF: Conselho Nacional do Meio Ambiente - CONAMA.

BORAH, R., et al. Contemporary distribution and impending mobility of arsenic, copper and zinc in a tropical (Brahmaputra) river bed sediments, Assam, India. Ecotoxicology and Environmental Safety, 161, p. 769-776, 2018. https://doi.org/10.1016/j.ecoenv.2018.06.038

CASADO-MARTíNEZ, M. C., BUCETA, J. L., BELZUNCE, M. J., DELVALLS, T. A. Using sediment quality guidelines for dredged material management in commercial ports from Spain. Environment International, v. 32, n. 3, p. 388-396, 2006. https://doi.org/10.1016/i.envint.2005.09.003

CHAKRABORTY, S., CHAKRABORTY, P., NATH, B. N. Lead distribution in coastal and estuarine sediments around India. Marine Pollution Bulletin, v. 97, n. 1-2, p. 36-46, 2015.

https://doi.org/10.1016/j.marpolbul.2015.05.056

CORRÊA, Iran Carlos Stalliviere. Aplicação do diagrama de pejrup na interpretação da sedimentação e da dinâmica do Estuário da Baía de Marajó-PA. Pesquisas em

Geociências, Porto Alegre, v. 32, n. 2, p.109-118, maio 2005. https://doi.org/10.22456/1807$\underline{9806.19551}$ 
EVANS, E. Hywel et al. Atomic spectrometry updates: review of advances in atomic spectrometry and related techniques. Journal Of Analytical Atomic Spectrom, Reino Unido, v. 29, n. 6, p.773794, 2014. https://doi.org/10.1039/c4ja90019g

FAIRBRIDGE, R. W. The encyclopedia of geomorphology. Encyclopedia of Earth Sciences Series. Vol. III. Dowden, Hutchinson e Ross Inc. Stroudsburg, p. 325-330, 1989.

FAPESPA- Fundação Amazônia de Amparo a Estudos e Pesquisas. Estatísticas Municipais Paraenses: Vigia. / Diretoria de Estatística e de Tecnologia e Gestão da Informação.Belém 62 f. n.1, jul/dez , 2016.

FERNANDES, Aldo Rafael Pascoal. Caracterização hidrodinâmica do Furo da Laura (Rio Guajará-miri), Amazônia Oriental. 2015. 45 f. Dissertação (Mestrado) - Curso de Geofísica, Programa de Pós Graduação em Geofísica, Universidade Federal do Pará, Belém, 2014.

FERREIRA, A. R.. Desenvolvimento inicial de teca (tectonagrandis) em resposta à fertilização com npk em diferentes condições edafoclimáticas no estado do Pará. 2015. 93 f. Dissertação (Mestrado) - Curso de Ciências Ambientais, Programa de Pós-graduação em Ciências Ambientais, Universidade Federal do Pará, Belém, 2015.

HARBISON, P. Mangrove muds-A sink and a source for trace metals. Marine Pollution Bulletin, v. 17, n. 6 , p. $246-250,1986$. https://doi.org/10.1016/0025-326X(86)90057-3

HAN, L., GAO, B., HAO, H., ZHOU, H., LU, J., SUN, K. Lead contamination in sediments in the past 20 years: A challenge for China. Science of The Total Environment, 746-756, 2018. https://doi.org/10.1016/j.scitotenv.2018.05.330

HOLANDA, N. S. Comportamento de metais pesados e nutrientes nos sedimentos de fundo da Baía do Guajará e Baía do Marajó. Dissertação (Mestrado em Geologia e Geoquímica) Universidade Federal do Pará (UFPA). Belém-PA, 2010.

KABATA-PENDIAS, Alina; PENDIAS, Henryk. Trace elements in soils and plants. 3. ed. United States ofAmerica: Crc Press, 2001. 331 p. https://doi.org/10.1201/9781420039900

KANBAR, H. J., et al. Iron mineralogy as a fingerprint of former steelmaking activities in river sediments. Science of The Total Environment, 540-553.

https://doi.org/10.1016/i.scitotenv.2017.04.156

KRAUSKOPF, Konrad Bates. Introdução a geoquímica II: tradução de Michal Landoe Paulo S. C. Bogus. São Paulo. Polígono, Editora USP, 1972.

KAUSHIK, A., KANSAL, A., SANTOSH, MEENA, KUMARI, S., \& KAUSHIK, C. P. Heavy metal contamination of river Yamuna, Haryana, India: Assessment by Metal Enrichment Factor of the Sediments. Journal of Hazardous Materials, v. 164, n. 1, p. 265-270, 2009.

https://doi.org/10.1016/j.jhazmat.2008.08.031

KEDE, Maria Luiza Félix Marques. Comportamento do chumbo em latossolos após aplicação de rocha fosfatada brasileira: subsídio para remediação ambiental de áreas contaminadas. 2006. 68 f. Dissertação (Mestrado) - Curso de Ciência, Centro de Informação Científica e Tecnológica, Escola Nacional de Saúde Pública Sergio Arouca, Rio de Janeiro, 2006.

LEMES, Marcos José da Lima. Avaliação de metais e elementos-traço em águas e sedimentos das bacias hidrográficas dos rios Mogi-Guaçu e Pardo, São Paulo. 2001. $215 \mathrm{f}$. Dissertação (Mestrado) - Curso de Ciências, Tecnologia Nuclear, Instituto de Pesquisas Energética e Nucleares, São Paulo, 2001. 
LIMA, E. A. R. de et al. Aplicação na técnica de infravermelho na identificação e caracterização da matéria orgânica nos sedimentos de fundo da Plataforma Continental do Amazonas. Geochimica Brasiliensis, Minas Gerais, v. 18, n. 1, p.46-53, 2004.

MARCHAND, C., LALLIER-VERGÈS, E., BALTZER, F., ALBÉRIC, P., COSSA, D., BAILLIF, P. Heavy metals distribution in mangrove sediments along the mobile coastline of French Guiana. Marine Chemistry, v. 98, n. 1, p. 1-17, 2006. https://doi.org/10.1016/j.marchem.2005.06.001

MENDES FILHO, N. E. Caracterização química da matéria orgânica nos sedimentos do solo do manguezal e dinâmica de nutrientes nas águas superficiais e intersticiais no médio estuário do rio Paciência em Iguaíba - Paço do Lumiar (MA). 2009, 162 F. Tese (Doutorado em Química Analítica) - Universidade Federal da Paraíba, João Pessoa, 2009.

MILESI, S. V. et al. Efeitos de Metais Cobre (Cu) e Zinco (Zn) sobre a comunidade de macroinvertebrados bentônicos em riachos do Sul do Brasil. Acta Scientiarum. Biological Sciences, Manringá, v. 30, n. 3, p. 283-289, 2008. https://doi.org/10.4025/actascibiolsci.v30i3.677

NEVES, Francisco de Assis. Fundamentos de limnologia. 2. ed. Rio de Janeiro: Interciência, 1998. $602 \mathrm{p}$.

OLIVEIRA, D. D., SOUZA-SANTOS, L. P., SILVA, H. K. P., MACEDO, S. J. Toxicity of sediments from a mangrove forest patch in an urban area in Pernambuco (Brazil). Ecotoxicology and Environmental Safety, 104, p. 373-378, 2014. https://doi.org/10.1016/j.ecoenv.2014.02.004

OLIVEIRA, E. C. et al. Distribuição dos metais traços em sedimentos de fundo do sistema hidrográfico da região de Belém, PA (margem oeste da baía do Guajará e rio

Carnapijó). Geochimica Brasiliensis, Belém, v. 29, n. 2, p.139-153, 2015.

PACHECO, José João. Governo do Estado do Pará. Estatística municipal: vigia. Pará: Instituto do Desenvolvimento Economico e Social do Estado do Pará, 2011. 48 p.

RAHMAN, M. S., et al. Source of metal contamination in sediment, their ecological risk, and phytoremediation ability of the studied mangrove plants in ship breaking area, Bangladesh. Marine Pollution Bulletin, 141, 137-146. 2019. https://doi.org/10.1016/..marpolbul.2019.02.032

RINKLEBE, J., SHAHEEN, S. M. Redox chemistry of nickel in soils and sediments: a review. Chemosphere, 179, 265-278, 2017. https://doi.org/10.1016/j.chemosphere.2017.02.153

RUBIO, B., NOMBELA, M.., VILAS, F. Geochemistry of Major and Trace Elements in Sediments of the Ria de Vigo (NW Spain): an Assessment of Metal Pollution. Marine Pollution Bulletin, v. 40, n. 11, p. 968-980, 2000. https://doi.org/10.1016/S0025-326X(00)00039-4

SANTOS, I. V. dos. Diagnóstico sobre as condições socioeconômicas e ambientais das comunidades no entorno do reflorestamento da empresa Pampa Exportações, Vigia de Nazaré, Pará, Brasil. 2012. 125 f. Dissertação (Mestrado) - Curso de Engenheira Agrônoma, Agriculturas Familiares e Desenvolvimento Rural., Universidade Federal do Pará, Belém, 2012.

SANTOS, R. S. B. Aspectos da hidrografia brasileira. Revista Brasileira de Geografia, n. 3, 327500, 1962.

SARTORI, Aderson; LOMBARDI NETO, Francisco; GENOVEZ, Abel Maia. Classificação Hidrológica de Solos Brasileiros para Estimativa da Chuva Excedente com o Método do Serviço de Conservação do Solo dos Estados Unidos Parte 1: Classificação. Revista Brasileira de Recursos Hídricos, v.10, n. 4, p. 05-18, out./dez. 2005. https://doi.org/10.21168/rbrh.v10n4.p5-18 
SCHAEFFER-NOVELLI, Yara; COELHO JUNIOR, Clemente. Onde vivem os manguezais e porque são tão importantes. In: INSTITUTO CHICO MENDES DE CONSERVAÇÃO DA BIODIVERSIDADE. Atlas dos Manguezais do Brasil. Brasília: Instituto Chico Mendes de Conservação da Biodiversidade, 2018. p. 176.

SILVA, Douglas Batista da. Qualidade de água e sedimento em reservatório. 2016. 170 f. Dissertação (Mestrado) - Curso de Ciências, Instituto de Pesquisas Energéticas e Nucleares, Universidade de São Paulo, São Paulo, 2016.

SIQUEIRA, G. W.et al. Determinação dos elementos-traço ( $\mathrm{Zn}$, Co e Ni) em sedimentos da Plataforma Continental Amazônica sob influência da descarga do rio Amazonas. Acta

Amazônica, Manaus. v.36, n.3, p. 321-326, 2006. https://doi.org/10.1590/S0044$\underline{59672006000300005}$

SIQUEIRA, G. W. et al. Determinação da matéria orgânica em sedimentos de fundo dos estuários de Santos/São Vicente e Baía de Santos-SP/Brasil. In: CONGRESSO BRASILEIRO DE PESQUISAS AMBIENTAIS E SAÚDE, 3., 2003. [Anais...]. p. 131-136.2003.

SIQUEIRA, G. W.; APRILE, F.. Avaliação de risco ambiental por contaminação metálica e material orgânico em sedimentos da bacia do Rio Aurá, Região Metropolitana de Belém - PA. Acta Amazonica, Manaus, v. 1, n. 43, p. 51-62, 2013. https://doi.org/10.1590/S0044-59672013000100007

SOUZA FILHO, P. W. M. Costa de manguezais de macromaré da Amazônia: cenários morfológicos, mapeamento e quantificação de áreas usando dados de sensores remotos. Revista Brasileira de Geofísica, Brasil, v. 23, n. 4, p. 427-435, 2005. https://doi.org/10.1590/S0102$\underline{261 \times 2005000400006}$

TOMAZELLI, A. C. Estudo comparativo das concentrações de cádmio, chumbo e mercúrio em seis bacias hidrográficas no Estado de São Paulo. 2003. 126 f. Tese (Doutorado) - Curso de Ciências, Biologia Comparada, Faculdade de Filosofia, Ciências e Letras, Ribeirão Preto, 2003.

WANG, J. et al. Tracking historical mobility behavior and sources of lead in the 59-year sediment core from the Huaihe River using lead isotopic compositions. Chemosphere, v. 184, p. 584-593. 2017. https://doi.org/10.1016/i.chemosphere.2017.06.022

ZAMBETTA, P. M. A. Espécies químicas inorgânicas (Al, As, Cd, $\mathrm{Cr}, \mathrm{Cu}, \mathrm{Fe}, \mathrm{Hg}, \mathrm{Mn}, \mathrm{Ni}, \mathrm{Pb}, \mathrm{e}$ Sn) no sedimento e nos sedimentos em suspensão do rio Corumbataí, SP. 2006. 73 f.

Dissertação (Mestrado) - Curso de Engenharia Agrônoma, Ecologia de Agrossistemas, Universidade de São Paulo, Piracicaba, 2006.

ZHANG, Z., et al. Physico-chemical forms of copper in water and sediments of Lake Pontchartrain basin, USA. Chemosphere, n. 195, p. 448-454, 2018.

https://doi.org/10.1016/j.chemosphere.2017.12.115 